\title{
Pondérer les espèces dans les formations herbacées très hautes
}

\author{
Ph. Daget ${ }^{1}$ T. S. Konaté 2
}

Mots-clés

Savane - Pâturage - Méthode

\section{Résumé}

Les méthodes d'inventaire classiques ne sont pas utilisables dans les formations herbacées très hautes (supérieures à $2 \mathrm{~m}$ ). Les auteurs proposent de faire $33 \mathrm{ob}$ servations par parcelle avec un quadrat en $U$ de $1 \mathrm{~m}$ de côté. Dans chacune, on fait la liste des espèces pondérée par une note d'abondance-dominance simplifiée de 0 à 3 . Les 33 notes sont additionnées pour obtenir la pondération définitive des espèces de la parcelle par des notes qui varient de 0 à 99.

\section{Difficultés rencontrées dans ces formations}

Une formation herbacée, ou une formation graminéenne, est considérée comme très haute lorsque sa hauteur moyenne est supérieure à $2 \mathrm{~m}(1)$. C'est le cas, en région tempérée, des roselières (Phragmites arundinacea) et aussi des cultures de mays (Zea mays) et parfois de tournesol (Helianthus annuus), en région méditerranéenne des cannes de Provence (Arundo donax) et en régions tropicales humides de nombreuses savanes à andropogonées.

Les méthodes de points-quatrat $(1,2)$ même modifiées par l'emploi des baionnettes $(1,2,5)$ ou de grandes aiguilles $(1,2,6)$ ne sont pas adéquates car elles supposent toutes que l'oeil de l'observateur se trouve au-dessus, ou, à la limite, au niveau de l'appareil, ce qui ne peut être le cas dans ces formations qui le surplombent nettement.

Les méthodes d'analyse de la voûte par photographies hémisphériques ne permettent pas les distinctions spécifiques. Elles ne répondent donc pas non plus au problème posé. Quant aux méthodes phytosociologiques, elles ne sont pas suffisamment précises pour suivre des évolutions ou des phénophases.

Aussi la mise au point d'un procédé d'approche nouveau était-il nécessaire.

\section{Le quadrat en $\mathrm{U}$}

L'emploi du quadrat en U (figure 1) est donc proposé (3, 4). Il correspond à un carré de $1 \mathrm{~m}$ de côté, trois de ces côtés étant matérialisés par des éléments constitués de profilé de fer en $\mathrm{T}$ dont le jambage est placé à l'extérieur. Ces éléments sont soudés solidement (à l'arc électrique) et leur extrémité libre est coupée en biseau de manière à permettre une introduction aisée dans les végétations denses.

La végétation en place est coupée sur une bande de $50 \mathrm{~m}$ de long et d'environ $1,5 \mathrm{~m}$ de large. L'observateur se place dans cette "allée" et a donc devant lui un "mur" de végétation. Il enfonce le

1. CNRS/CIRAD-EMVT, Campus international de Baillarguet, BP 5035, 34032 Montpellier Cedex 1, France

2. Direction de l'hydraulique pastorale, BP 500, Bamako, Mali

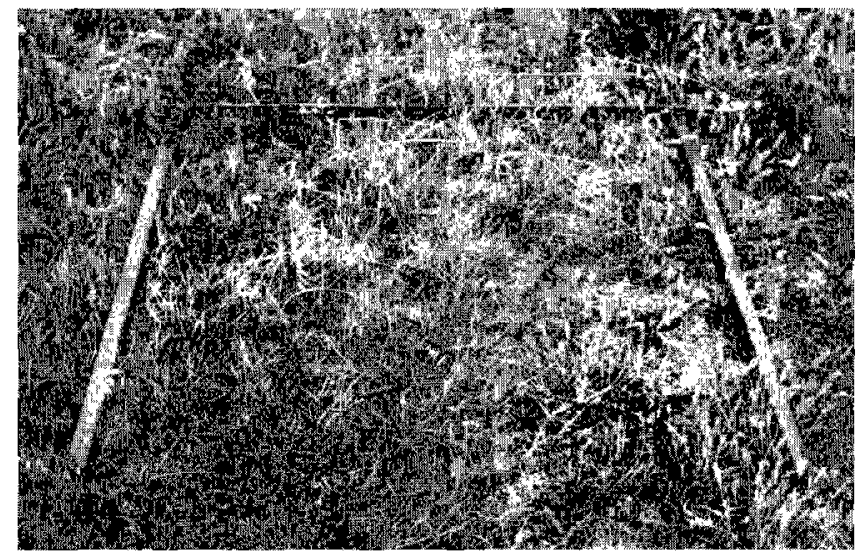

Figure 1 : le quadrat en $U$ dans une végétation herbacée basse.

quadrat en $U$ dans la végétation jusqu'à ce que sa base arrive au ras du "mur" de végétation (figure 2). Il peut alors procéder aux observations proprement dites. La même manœuvre est effectuée 33 fois, en déplaçant le quadrat de manière à laisser un espace de $50 \mathrm{~cm}$ entre deux positionnements successifs.

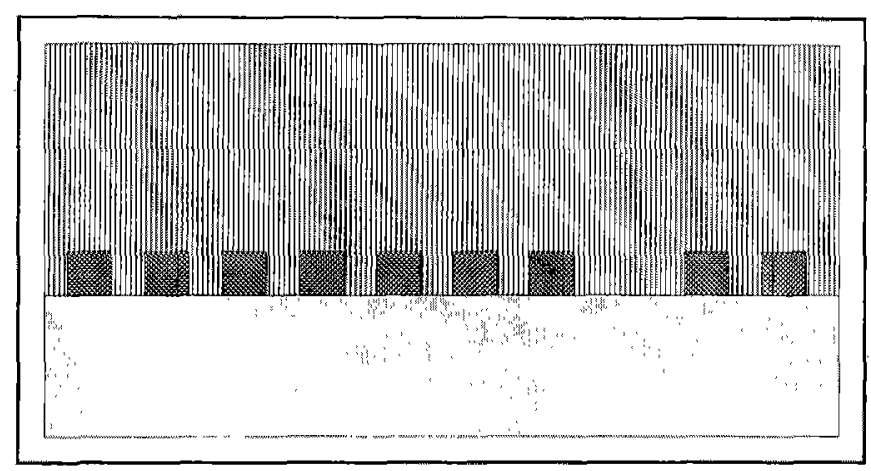

Figure 2 : disposition des quadrats en $U$ dans la végétation. Rayures: la végétation de la savane intacte ; grisé : partie coupée de cette végétation; croisillons : position des 7 premiers quadrats et des 2 derniers. 
Dans chaque quadrat, les observations sont faites de la manière suivante :

- la liste des espèces dont la souche ou le collet est dans le quadrat, ou dont le houppier se trouve au dessus, est établie ;

- chaque espèce de la liste est ensuite pondérée par une note qui est :

- note 0 : si l'espèce y est très rare, avec un seul pied ou un recouvrement au plus égal à $1 / 4 \mathrm{~m}^{2}$;

- note 1 : si l'espèce est peu abondante, ou son recouvrement est de l'ordre de $1 / 2 \mathrm{~m}^{2}$;

- notc 2 : si l'espèce cst abondante, ou son rccouvrement est de l'ordre de $3 / 4$ de $\mathrm{m}^{2}$;

- note 3 : si l'espèce est très abondante, ou présente un recouvrement supérieur à $3 / 4$ de $\mathrm{m}^{2}$.

La liste complète des espèces présentes correspond à la fusion des 33 listes élémentaires ; chaque espèce est pondérée par la somme des notes qui lui ont été données dans les quadrats élémentaires. La liste des espèces de la station est ainsi établie et assortie d'une note variant entre 0 et 99 , note qui peut être assimilée à la mesure d'un recouvrement et utilisée comme telle. S'il est jugé souhaitable de distinguer les espèces très rares de celles qui sont totalement absentes, on pourra remplacer la note zéro par une faible valeur décimale : 0,1 .

Ainsi la "complexion" : 10 fois peu abondante - 10 fois rare - 13 fois absente sera distinguée de 10 fois peu abondante et 23 fois absente par : $11=(10 \times 1)+(10 \times 0,1)+(13 \times 0)$ contre $10=(10 \times 1)+(23 \times 0)$.

\section{Summary}

Daget Ph., Konaté T. S. Weighing species in tall grass vegetation

Usual methods of survey cannot be used in tall grass vegetation (over $2 \mathrm{~m}$ high). The authors recommand that 33 quadrats be analysed with a $1 \mathrm{~m} \times 1 \mathrm{~m} U$-frame. For each one of them a list of the species is written up, whose abundance-dominance weight is given by using a simplified grade ranging from 0 to 3 . For each species, the 33 grades are added in order to obtain the final weight grade ranging from 0 to 99 .

Key words: Savanna - Grazing - Method.

\section{BIBLIOGRAPHIE}

1. DAGET Ph., POISSONET J., 1991. Prairies permanentes et pâturages. Méthodes d'étude. Montpellier, France, Institut de Botanique, 375 p.

2. DAGET Ph., POISSONET J., 1971. Une méthode d'analyse phytologique des prairies, critères d'application, Anm. Agron., 22 (1) : 5-41.

3. DAGET Ph., GASTON A., 1994. SUIVI : Un logiciel pour l'analyse diachronique de la production primaire des pâturages au Mali. Montpellier, France, CIRAD-EMVT, 13 p.

4. KONATE T., 1994. Agrométéorologie appliquée à la surveillance des ressources pastorales au Mali. Montpellier, France, CIRAD-EMVT, 59 p. et annexes.

5. POISSONET P., DAGET Ph., POISSONET J., LONG G., 1971. Rapid point survey by bayonet blade. J. Range Managc., 25 (4) : 313.

6. POISSONET J., CESAR J., 1972. Structure spécifique de la strate herbacée dans la savane à Palmier Rônier de Lamto, Côte d'lvoire. Ann. Univ. Abidjan, F 5 : 577-601.

Reçu le 6.10.95, accepté le 5.4.96

\section{Resumen}

Daget Ph., Konaté T. S. Ponderar las especies en las formaciones herbáceas muy elevadas

Los métodos de inventario clásicos no son indispensables en las formaciones herbáceas muy elevadas (de más de $2 \mathrm{~m}$ ). Los autores proponen realizar 33 observaciones por parcela, con un cuadriculado en $U$, de un metro de cada lado. En cada una, se hace una lista de especies, ponderada por una nota de abundancia-dominancia simplificada de 0 a 3 . Las 33 notas se adicionan para obtener la ponderación definitiva de las especies de la parcela por medio de notas que varían de 0 a 99 .

Palabras clave : Sabana - Pastoreo - Método. 